\title{
Artifact Removal in Physiological Signals-Practices and Possibilities
}

\author{
Kevin T. Sweeney, Student Member, IEEE, Tomás E. Ward, Senior Member, IEEE, \\ and Seán F. McLoone, Senior Member, IEEE
}

\begin{abstract}
The combination of reducing birth rate and increasing life expectancy continues to drive the demographic shift toward an aging population. This, in turn, places an ever-increasing burden on healthcare due to the increasing prevalence of patients with chronic illnesses and the reducing income-generating population base needed to sustain them. The need to urgently address this healthcare "time bomb" has accelerated the growth in ubiquitous, pervasive, distributed healthcare technologies. The current move from hospital-centric healthcare toward in-home health assessment is aimed at alleviating the burden on healthcare professionals, the health care system and caregivers. This shift will also further increase the comfort for the patient. Advances in signal acquisition, data storage and communication provide for the collection of reliable and useful in-home physiological data. Artifacts, arising from environmental, experimental and physiological factors, degrade signal quality and render the affected part of the signal useless. The magnitude and frequency of these artifacts significantly increases when data collection is moved from the clinic into the home. Signal processing advances have brought about significant improvement in artifact removal over the past few years. This paper reviews the physiological signals most likely to be recorded in the home, documenting the artifacts which occur most frequently and which have the largest degrading effect. A detailed analysis of current artifact removal techniques will then be presented. An evaluation of the advantages and disadvantages of each of the proposed artifact detection and removal techniques, with particular application to the personal healthcare domain, is provided.
\end{abstract}

Index Terms-Adaptive Filter, artifact removal, blind source separation (BSS), canonical correlation analysis (CCA), independent component analysis (ICA), Kalman filter, personal healthcare, Wiener filter.

\section{INTRODUCTION}

$\mathbf{O}$ VER the past few decades, hospital patient numbers have increased globally, placing an increased burden on healthcare systems. This growth in numbers is, in part, due to increased life expectancy and the rise in chronic illnesses. The increased burden is reflected in the growth of annual healthcare expenditure of national governments. Expenditure on national healthcare has risen dramatically in both the U.S. and in most countries in the European Union (EU). For the member states of the EU included in a study conducted by the Ministry of Health

Manuscript received August 1, 2011; revised November 18, 2011 and January 5, 2012; accepted February 9, 2012. Date of publication February 22, 2012; date of current version May 4, 2012. This work was supported in part by Irish Research Council for Engineering, Science and Technology (IRCSET).

The authors are with the Department of Electronic Engineering, National University of Ireland, Maynooth, Ireland (e-mail: ksweeney@eeng.nuim.ie; tomas.ward@eeng.nuim.ie; sean.mcloone@eeng.nuim.ie).

Digital Object Identifier 10.1109/TITB.2012.2188536 and Social Affairs in Sweden, it has risen from an average of $3.1 \%$ of the gross domestic product (GDP) in 1960 to $8.8 \%$ in 2006 [1]. In the U.S., healthcare was costing eight times more in 2008 than in 1980 and accounted for $16.2 \%$ of the nations GDP [2]. This increase in national expenditure is unsustainable and a more cost-effective delivery of health services is an imperative. Recent advances in information and communications technology (ICT) have enabled some of the more rudimentary examinations performed during in-patient care to be performed in an out-patient, personal healthcare domain. This ability to monitor the patient in the comfort of their own home can greatly improve quality of life, with the added benefit that out-patient care can cost as little as 50\% of their inpatient counterparts [3], thus, making them a more feasible option in many instances.

In-home monitoring allows the acquisition of patient information during activities of normal daily living, resulting in more representative data over longer time frames and less inconvenienced patients. The added freedom for the patient can, however, come at a cost to the acquisition of clean useful data as the in-home environment is poorly controlled for extraneous sources of measurement noise (normally tightly controlled in a hospital setting) and the lack of technical oversight to ensure the measurement procedure is properly maintained at all times.

Consequently, the development of algorithms capable of removing or at least identifying artifacts in the recorded data are critically important. The term artifact has different definitions in different application domains. In the context of this paper, artifacts are defined as any undesired variation in the measured signal due to sources external to the parameter of interest.

Personal healthcare systems for in-home monitoring are generally designed to operate for long periods without recharging and to be inexpensive as patients may lose, damage or neglect to return the device. This goal of a cheap, reliable, energy-efficient device results in robust systems with limited computational capability limiting the complexity of the signal processing algorithms that can be incorporated. In this paper, we review current techniques employed for physiological signal artifact removal, with a particular emphasis on those signals and techniques that can be employed in a personal healthcare setting. Section II describes biosignals most often recorded during clinical evaluation. Next, Section III gives a brief explanation of the most common artifact types detected in physiological signal recordings. In Section IV, the most common artifact removal techniques currently utilized in signal processing are described. Finally, Section $\mathrm{V}$ reviews the factors that impact on the choice of artifact removal algorithm and discusses the challenges for future development in this area. 


\section{MEASUREMENT TECHNIQUES}

There are many clinically relevant signals that can be measured from the human body such as body temperature, $\mathrm{pH}$ levels [4], and perspiration levels [5]. These signals tend to vary slowly over time and as such do not warrant continuous monitoring. The primary physiological measurements of diagnostic utility that require more regular monitoring are electrical or optical in nature and this sets the character of the artifacts typically encountered during their acquisition.

Electrocardiography (ECG) is the well-known electrical measurement of cardiac activity recorded externally by skin electrodes. The set of signals acquired during ECG are used by clinicians to ascertain a patient's cardiovascular health. The transitory appearance or absence of specific features in the cardiac waveform can be directly related to parameters of physiological importance [6] necessitating high quality, continuous, long duration monitoring during activities of normal daily living [7], [8], [9].

Electromyography (EMG) is a technique for evaluating skeletal muscle contraction [10] and is largely used in movement science studies and rehabilitation medicine [11]. For example, Azzerboni et al. [12] used long-term EMG recordings to differentiate between parkinsonian and essential tremor and correctly diagnosed in $93 \%$ of their cases. The growth of wearables [13] in a personalized-health context has led to the incorporation of EMG sensors for a broader variety of uses; for example, monitoring of trapezius muscle activity is used in [14] as an indication of physiological stress. Another major application of EMG recordings is in the control of prosthesis [15], [16]. By utilizing the available functional muscles in the body, a subject is capable of controlling an inactive muscle group or limb through the use of prosthesis. All the uses of EMG stated earlier, along with many others, require long-term stable operation necessitating the need for good quality measurement in an external environment.

Electroencephalography (EEG) is the recording of electrical activity, produced by the firing of neurons within the brain [17] and has a wide range of functions in modern medicine. One of the most common uses is in the area of epilepsy [18]. Epileptic activity can be very hard to measure due to the uncertainty of the exact time an event will occur. Therefore, long-term monitoring is often employed to increase the probability of detection. This long-term monitoring can cause discomfort to the patient who must remain in a motion-free state for an extended period of time. The recording of the EEG in an in-home, personal healthcare, domain can increase the users level of comfort and allow for longer recording durations, while still recording the data to a satisfactory level. Other uses of EEG include the detection of sleep/drowsiness levels [19], [20], [21], cognitive workload [20], [22] and in brain-computer interfaces (BCI) [23], [24], [25], [26] all of which have applications in a personal healthcare domain.

Photoplethysmography (PPG) is an optically obtained volumetric measurement of an organ or tissue [27]. Simple and inexpensive, it is often used to monitor heart rate and other parameters of the cardiac cycle as well as respiration [28]. Pulse oximetry $(\mathrm{POX})$ is a related technique using spectroscopic methods to derive a measure of arterial oxygen saturation $\left(\mathrm{SpO} \mathrm{O}_{2}\right)$. This quantity responds rapidly to hypoxemic events and is very simple to interpret [29]. It is often used in situations, where there is a need to monitor a patients oxygenation levels, for example, in intensive care, critical care, and emergency department areas of a hospital. However, it can also be utilized in a personal healthcare environment to supervise recovery after heart problems and to aid the diagnosis of some sleep disorders such as apnea and hypopnoea [30].

A more sophisticated variation of POX is functional near infrared spectroscopy (fNIRS) which uses the same principles to measure the changing oxygen levels in the brain. fNIRS employs light at different wavelengths (in the infrared band) to penetrate the skull, and to indirectly measure the change in the concentration levels of oxygenated (oxy-Hb) and deoxygenated hemoglobin (deoxy-Hb) in the brain, through changes in the optical absorption [31]. The fNIRS technique detects the activation changes in the cortex, allowing optical measurements to be used for imaging brain functions [32].

Medical applications of fNIRS center on the noninvasive measurement of the quantity and oxygen content of hemoglobin. These measurements are then used in the determination of cognitive tasks [33] and in BCI [34]. At present, the high cost of fNIRS systems makes them less suitable for nonclinical settings, but as the systems continue to be refined and become more affordable they are becoming a more viable option for in-home personal healthcare.

Respiration is the transport of oxygen from the air in the environment to the cells within tissues and the transport of carbon dioxide in the reverse direction. The recording of respiration is important in the evaluation of a patient's well being. Medical studies also suggest the volume of air inhaled per minute at rest $(6 \mathrm{~L} / \mathrm{min})$, greatly increases when a patient is unhealthy (Heart disease $12( \pm 3) \mathrm{L} / \mathrm{min}$ [35], Diabetes $15( \pm 2) \mathrm{L} / \mathrm{min}$ [36], and Cystic Fibrosis 11-14 L/min [37]), thus, providing a further rationalism for the need for respiration measurements. The detection of sleep apneas often requires the patient to be present in a hospital for overnight respiratory monitoring. Using in-home measurement systems allows for the same respiratory experiments to be undertaken without the need for a hospital visit [38]. A number of different methods used to record respiration rate are discussed in Tarrent et al. [39].

In the next section, we highlight in detail the etiology of the most common artifacts present in such a measurement domain and how these manifest in the instrumentation task.

\section{ARTIFACTS}

The presence of artifacts seriously degrades the clinical utility of measurements taken in a home environment. There are three distinct sources of artifacts that impact all physiological recordings; environmental artifacts, experimental errors, and systemic physiological artifacts [32]. These three sources have different properties and, thus, manifest themselves distinctively on the measured signals. 


\section{A. Environmental Artifacts}

Environmental artifacts primarily originate from the mains power leads that surround the body during day to day living and can be seen in the form of $50 / 60 \mathrm{~Hz}$ hum. It can additionally arise from electromagnetic interference (EMI); a disturbance that affects the signal due to the electromagnetic radiation emitted from an external source. This EMI can interact with the measurement cables, or the human body which acts as an antenna to the RF interference. Instrumentation artifact is a type of environmental artifact which is said to originate from within the circuit components themselves and can be observed in the form of thermal noise (due to the changing temperature of components), shot noise (due to the quantized nature of the electric charge) or $1 / f$ (pink) noise (due to the slow fluctuations of the condensed-matter materials of the devices). Environmental artifact is a major source of interference in bioelectric measurements due to the capacitive coupling of the measurement cables with the mains [40] and other devices.

The recording leads can also be susceptible to magnetically induced interference, but this can be reduced by shortening the lead lengths and twisting the cables so both receive the same interference. Further, the recording leads are also shielded to avoid RF interference and capacitative coupling. Optical measurement devices, such as PPG and NIRS, are not intrinsically susceptible to capacitive coupling interference as no bioelectric measurement is conducted. However, they are sensitive to environmental artifacts from other natural and artificial light sources. These interferences can be reduced by using correct procedure and ensuring that no external lighting can affect the recording.

Artifacts from environmental sources can normally be suppressed by means of a simple filter. This is due to either the narrow frequency band of the artifact $(50 / 60 \mathrm{~Hz}$ hum) or because the frequency band that the artifact occupies is not in the same range as the desired signal. Although white noise is a broadband signal covering all frequencies, filtering the undesired high-frequency components removes the majority of the noise, thereby improving the signal-to-noise ratio (SNR).

\section{B. Experimental Error}

Experimental error is seen as uncontrolled/unwanted variation in the experimental setup. Experimental error can be reduced by proper procedure and planning, but it is almost impossible to eliminate completely. This inability to completely eradicate the error is, in most part, due to human error during experimental setup or subject motion during data acquisition. Incorrect procedural setup, for example, the poor application of the electrodes, can create discrepancies in the measured signal. In the personal healthcare environment, the user is not always a trained technician, and thus the correct procedure in preparation and recording may not always be adhered to. However, these errors can often be observed prior to recording and, thus, corrected. Motion of the subject can create a large amount of experimental error and is commonly the most detrimental for many physiological signal recordings, due to changes in the underlying measurement geometry. This source of error, although present in most recordings, is far more prominent in the personal healthcare environment due to the patient having more freedom of movement. Subject motion can also cause changes to the coupling between the sensors and the tissue surface introducing further distortion to the recorded signals.

With respect to optical methods, PPG and fNIRS for example, motion of the subject causes two distinct types of artifact. First, movement of the source or detector optodes with respect to the skin causes a change in the path length taken by the light transmitted by the source optode before it enters the detector. Subject movement also causes a blood pooling effect observable in the measurements. This blood pooling creates an increase in the overall blood levels at the recording site [41] and disrupts the ability to resolve the functional induced change.

Motion also has a damaging effect on biopotential measurements in the body, such as ECG and EEG. Subject motion can cause the position of the electrode on the skin to alter. This movement can cause a variation in the distance between the recording electrode and the skin, which results in a corresponding change in the electrical coupling, causing signal distortion. Subject motion can also cause a change in the conduction volume between the electrode and the signal source which induces potential changes at the recording sites [42]. These changes culminate in the production of artifact on the recorded output. The movement of the recording devices with respect to the underlying skin can be reduced by correct preparation of the interface surface by abrasion and cleansing of the skin prior to the adhesion of the electrodes [43].

Although the majority of breathing measurement devices monitor chest movement to indirectly detect the volumetric change of the lungs, they can equally be affected by undesired motion of the subject. This undesired motion is due to twisting or flexing of the torso. Care must, therefore, be taken to ensure the measurement device is situated in a position that reduces the probability of nonbreathing related movement.

Experimental artifacts, particularly those relating to motion, in the recorded signals are more difficult to remove than instrumental artifact as they generally do not have a predetermined narrow frequency band and their spectrum often overlaps that of the desired signal [44], and thus classical filtering approaches cannot be implemented.

\section{Physiological Artifact}

Physiological artifacts are variations in the desired signal due to other physiological processes in the body. Major contributors of artifacts most commonly detected in physiological measurements are eye movement-related artifacts, cardiac signals and muscle tension signals measured using EMG. Other physiological signals, such as brain signals, do not have sufficient signal strength to cause major complications to the desired measurements.

Eye movement-related artifacts have the largest detrimental effect on EEG. As the eye alters position, the resting potential of the retina changes and can be measured using an electrooculogram (EOG). Blinking also causes involuntary movement of the retina as well as muscle movements of the eye lid. Due to 
the eyes' close proximity to the brain, as the signal propagates over the scalp, it can appear in the EEG as an artifact that can present serious complications in EEG analysis [45]. The amplitude of these signals can be many times greater than the EEG signals of interest. This ocular signal can easily be measured using electrodes placed above and below the eye.

Muscle movements can cause artifacts on a broad range of electrophysiological signals as they can originate from any muscle on the body, and thus can be in very close proximity to the signal recording site. In terms of EEG recording, the tension of the facial or jaw muscles has a large detrimental effect on the desired signal. EMG does not, however, have an adverse effect on the signals recorded using light, for example, fNIRS and PPG, due to the different recording modalities.

The cardiac signal can cause artifacts on a number of different recordings including EEG and EMG. The electrical signal outputted from the heart has a high signal strength and so can be picked up at numerous positions around the body. The beating of the heart also causes pulse artifacts, where the expansion and contraction of the vessel introduce voltage changes into the recordings [46]. These signals have a frequency range of around $0.5-40 \mathrm{~Hz}$ and $1.2 \mathrm{~Hz}$, respectively, making it difficult to remove them from most physiological signals, as these are also located at these ranges.

Due to the overlapping spectra of the various physiological signals, the vast majority of artifacts that can be embedded on a signal have to be removed in a way that does not involve basic filtering to minimize data loss. In the following section, we review a number of the most commonly used algorithms available at present for artifact removal.

\section{ARtifact Removal TechniQues}

There are a number of general techniques used for artifact separation and removal. This separation can often be accomplished using simple classical filtering techniques, such as low-pass filtering. However, this can only be employed when the frequency bands of the artifact and the desired signal do not overlap. When there is spectral overlap, alternative techniques must be adopted.

Fig. 1 presents the general form of the artifact suppression techniques discussed in this review. The signal recorded during an experiment $\left(\mathbf{x}(n) \in \mathbb{R}^{P}\right)$ is a combination of the original desired signal $\left(\mathbf{s}(n) \in \mathbb{R}^{M}\right)$ contaminated with artifact $\mathbf{v}(n)$. The function $f$ is used to describe how the two signals are combined to produce the signal at the recording site. This allows for both multiplicative [47] and additive artifacts; however, additive artifacts are predominantly assumed as this leads to tractable problem formulations, i.e.

$$
\mathbf{x}(n)=\mathbf{s}(n)+\mathbf{v}(n) .
$$

Therefore, Fig. 1 evolves to Fig. 2. Label (i) depicts when a reference signal $\mathbf{u}(n)$ is used to estimate $\mathbf{v}(n)$ (for example, adaptive filtering) and (ii) depicts where $\mathbf{v}(n)$ is estimated directly from $\mathbf{x}(n)$ (for example, blind source separation (BSS) techniques). While both (i) and (ii) can be employed together, in practice, the methods developed fall into one or other of (i) or (ii). The majority of artifact removal techniques also assume

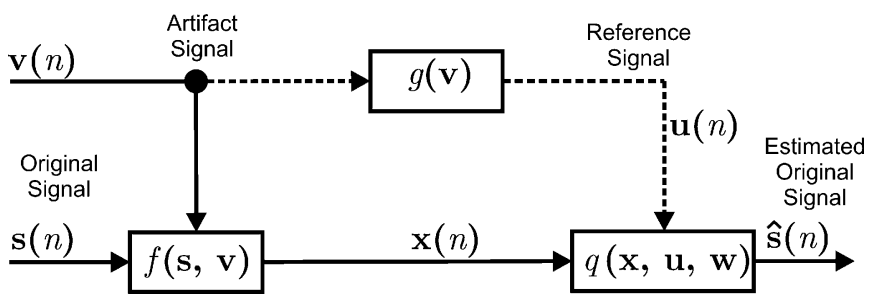

Fig. 1. General framework for artifact removal.

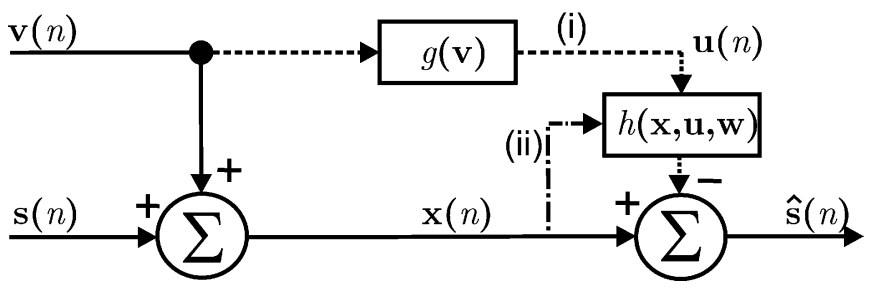

Fig. 2. Linear framework for artifact removal.

that the number of recorded signals must be equal to or larger than the number of sources, i.e., $P \geq M$. Section IV-E describes algorithms that operate when this is not the case.

The purpose of the numerous artifact removal techniques described in this section is to adapt the filter parameters $\mathbf{w}$ so as to minimize the mean square error between the output $\hat{\mathbf{s}}(n)$ and the desired original signal $\mathbf{s}(n)$, i.e.

$$
\min _{\mathbf{w}}\|\mathbf{s}(n)-\hat{\mathbf{s}}(n)\|^{2} .
$$

A description of some of the main techniques and algorithms currently employed in physiological signal artifact removal now follows.

\section{A. Adaptive Filtering}

Adaptive filtering operates under the assumption that the desired signal $s(n)$ and the artifact $v(n)$ are uncorrelated, i.e.

$$
E[s(n) v(n)]=0
$$

where $E[$.$] is the expectation operation. Using a tapped delay$ line, the filter generates a signal $\hat{v}(n)$ which is correlated with the actual artifact signal $v(n)$ from a reference signal $u(n)$. This estimate of the artifact is then subtracted from the recorded signal $x(n)$ and the residual $\hat{s}(n)$ is an estimate of the original signal $s(n)$ [48]. The filter coefficients continue to adapt until the artifact in the output, which is correlated with the provided reference, has been minimized.

1) References: The choice of artifact reference $u(n)$ is very important when utilizing an adaptive filter. For motion artifact, accelerometers are predominantly selected [49], [50] due to their high sensitivity level combined with an ease of application; however, other sources such as anisotropic magnetoresistive (AMR) sensors [51], optical bend sensors, impedance sensors [52] and skin stretch sensors [53] have also been considered.

References for artifacts originating from undesired electrophysiological signals can often be recorded using general recording techniques. Examples of this include the recording of EOG for the removal of eye movement artifact from 


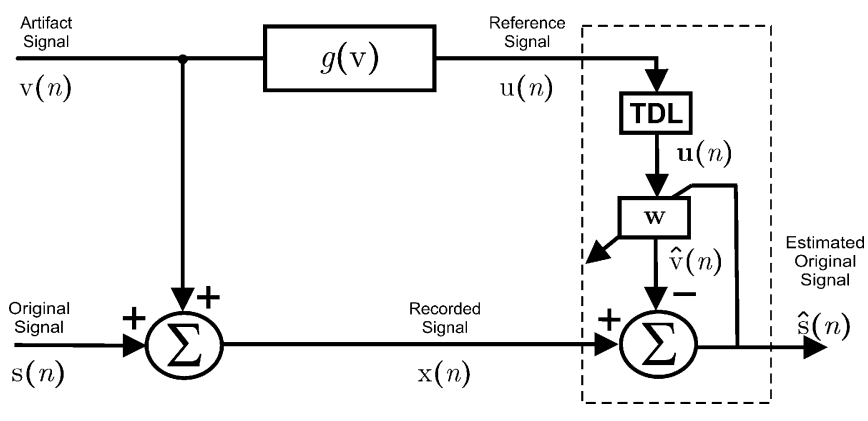

Fig. 3. Adaptive filter framework for artifact removal.

EEG [45] and EMG recording for removing muscle movement artifact from ECG [54]. However, other methods have also been proposed which develop a model of the artifact to use as a reference [44]. These model based methods can, however, add unnecessary complexity to the system and are not often implemented.

2) Algorithms: The choice of algorithm dictates the computational cost and accuracy of the adaptive filter. The most common algorithm employed in adaptive filtering is the least mean-square (LMS) algorithm with a computational complexity of $\mathrm{O}(L)$ ( $L$ is the filter length) [55] and which has a weight update equation [56]

$$
\begin{aligned}
\hat{s}(n) & =x(n)-\mathbf{w}^{T}(n) \mathbf{u}(n) \\
\mathbf{w}(n+1) & =\mathbf{w}(n)+2 \mu \hat{s}(n) \mathbf{u}(n) .
\end{aligned}
$$

This algorithm produces an estimate of the desired signal $\hat{s}(n)$ through adaption of the filter coefficients $\mathbf{w}(n)$. The filter coefficients are adjusted so as to minimize the mean squared amplitude of the estimated signal. The step size $\mu$ controls the rate of adaption and hence the overall stability of the filter.

Another family of algorithms commonly used in adaptive filtering is based on the recursive least square (RLS) algorithm. This is a computationally more complex algorithm with a computational complexity of $\mathrm{O}\left(L^{2}\right)$ [55] and an update equation [57]

$$
\begin{aligned}
\mathbf{G}(n) & =\frac{\mathbf{R}^{-1}(n-1) \mathbf{u}(n)}{\lambda+\mathbf{u}^{T}(n) \mathbf{R}^{-1}(n-1) \mathbf{u}(n)} \\
\mathbf{R}^{-1}(n) & =\lambda^{-1} \mathbf{R}^{-1}(n-1)-\mathbf{G}(n) \mathbf{u}^{T}(n) \lambda^{-1} \mathbf{R}^{-1}(n-1)
\end{aligned}
$$

$$
\mathbf{w}(n)=\mathbf{w}(n-1)+\hat{s}(n) \mathbf{G}(n)
$$

where $\mathbf{R}(n)$ is an estimate of the input signal covariance matrix and $\lambda$ is a forgetting factor. The RLS algorithm exhibits extremely fast convergence due its second-order nature. Many papers describe the advantages of both families of algorithms including others such as least mean absolute value and sign (SIGN) [58]. The RLS family of algorithms consistently performs to a higher accuracy than the other algorithms but in doing so have a higher computational cost [50], [59]. In [58] the authors tested five different algorithms from the two families of algorithms and found that the RLS algorithms achieved the highest accuracy (computed using correlation values) in 23 of the 28 computed tests, when removing ECG artifacts from
EMG recordings. This was especially true when the signal was assumed stationary and a simplified formulation was used. In 2009, Zia Ur Rahman et al. [54] used a normalized signed LMS algorithm in the cancelation of simulated artifacts on an ECG signal. This algorithm was found to converge faster than the basic LMS algorithm, had a lower computational cost and also had a greater SNR improvement ( $3.9 \mathrm{~dB}$ versus $9.2 \mathrm{~dB}$ in some cases), all of which are desired for operation in the personal healthcare domain.

The adaptive filter is easy to implement and it also has an ability to operate on-line and without preprocessing or calibration, but the requirement of additional sensors to provide reference inputs can add to the complexity of the hardware system.

\section{B. Wiener Filtering}

Wiener filtering is another parametric technique used to remove unwanted artifacts from the measured signals [60]. The Wiener algorithm is based on a statistical approach and thus does not require the use of an external reference signal. The signal and the (additive) artifact are assumed to be stationary linear stochastic processes with known spectral characteristics or known autocorrelation and cross-correlation. The desired signal and artifact are also assumed to be uncorrelated with each other.

The purpose of the Wiener filter is to produce a linear time invariant filter so that the mean square error between the true desired signal $s(n)$ and the estimated one $\hat{s}(n)$ is minimized [61] as stated in (2). This filter is determined using the power spectral densities (PSDs) of the signal and the artifact as described in [61]. These PSD are generally not available a priori and must be estimated from measurements.

The necessity for calibration prior to usage, and its inability to be employed in realtime applications, is a disadvantage of the Wiener filter with respect to the adaptive filter. However, it eliminates the requirement for additional hardware on the recording device necessary with the adaptive filter. In 2005, Izzetoglu et al. [61] used both adaptive filtering and Wiener filtering individually to try to remove motion artifact from recorded NIRS data. After calibration of the Wiener filter, the output was discovered to have a better SNR than that achievable using the adaptive filter (average $\Delta \mathrm{SNR}=3.25 \mathrm{~dB}$ determined using epochs of clean data as the true signal) demonstrating the advantage of the Wiener filter.

\section{Bayes Filters}

Bayesian filtering is a method of probabilistically estimating a given systems state from available noisy observations. Bayes filters express the state at the current time sample as $\mathbf{x}(n)$. The probability distribution over these random variables $\mathbf{x}(n)$ [known as belief, $\operatorname{Bel}(\mathbf{x}(n))]$ is a representation of the uncertainty. The Bayes filters operate by estimating the beliefs over the state space using all the available information encompassed in the observed data [62], using the assumption that the dynamic system is Markov. Markov systems assume that the current state variable $\mathbf{x}(n)$ contains all the relevant information about the system. 
Bayes filters are implemented using a predictor-corrector methodology. The predictor uses a time update model to describe the relationship between the states from one time sample to the next. The corrector step then utilizes a measurement model to describe the relationship between the observed data and the internal state.

This approach overcomes some of the limitations of adaptive filtering and Wiener filtering. Like Wiener filtering, the Bayes filter technique does not require a reference signal to be capable of removing the embedded artifact. However, unlike the Wiener algorithm, Bayes filtering is capable of operating in real time.

There are a number of different methods that implement the approximations of the Bayes filter technique currently utilized in signal processing. The Bayes filter technique is not itself implemented due to its algorithm being computationally intractable. Two of these methods are detailed as follows.

1) Kalman Filtering: Kalman filtering was first described by Kalman in 1960 [63]. The Kalman filter essentially implements a mathematical predictor-corrector type estimator as stated earlier. The filter uses feedback control to estimate a process: the filter first estimates the process state at a given time and then obtains feedback in the form of (noisy) measurements [64]. This creates two layers of calculations: time update equations and measurement update equations as detailed by Welch and Bishop in [64].

After each time and measurement update pair have completed, the process is repeated with the previous a posteriori estimate used to predict the new a priori estimate [64]. The filter models must be created prior to implementation of the algorithm. These models can be determined using a number of different modeling methods, such as autoregressive and moving average models. The requirement that the algorithm be calibrated before use [65] could lead to problems in the personal healthcare environment.

There are a number of approaches for achieving artifact removal using a Kalman filter. First, a model of both the desired signal and the contaminating artifact can be produced. The recorded signal can be described as the summation of these two model signals and, thus, the process and measurement models can be determined. The Kalman filter can then be implemented to estimate the unknown system parameters by reducing the variance of the error between the recorded output and the modeled output. With the system parameters now known, artifact removal can be accomplished by setting the parameters affiliated with the artifact in the measurement model to zero. This method was implemented by both Seyedtabaii et al. [66] and Morbidi et al. [67] to remove motion artifact and transcranial magnetic stimulation (TMS)-induced artifact from PPG and EEG signals, respectively, with promising results. A second method models just one of the combined signals, either the desired true signal or the contaminating artifact. Using this model, the Kalman update equations are then produced. Again the Kalman filter update equations are employed and the desired signal can be determined either as the function of the state space equation or as the residual [65], [68]. Reference signals can be used as an aid in the modeling of the signals [66], if available. The Kalman filter has also been implemented as part of an adaptive filtering scheme in [69], in which the Kalman filter update equations were employed to update the tap weights of the adaptive filter.

The Kalman filter operates on the assumption that the initial uncertainty (belief) is Gaussian and that the system dynamics are linear functions of the state [62]. However, as most systems are not strictly linear, the Kalman filter has also been extended to the nonlinear domain through the extended Kalman filter (EKF) [64]. This EKF is capable of performing to the same degree by linearizing around the current mean and covariance. The main advantage of the Kalman filter is in the computational efficiency of the algorithm due to its efficient use of matrix operations. However, the filter can only function with unimodal distributions which can be a problem in biomedical applications.

In 2001, Rohalova et al. [70] presented results showing the advantage of the EKF in the detection of artifacts in sleep EEG. Their results show that for that application, the nonlinear Kalman filter has a sensitivity of almost twice that of the linear Kalman filter. The EKF was also utilized by Sameni et al in 2005 [71] and 2007 [72] with simulated ECG signals. Here, the filter was used to remove environmental and muscle artifact from noisy ECG showing positive results with a $\triangle$ SNR ranging from 8.5 to $0.8 \mathrm{~dB}$ depending on the input SNR.

If the process and measurement models are available for the required system, the Kalman filter stands as a very attractive alternative to the adaptive filter for two major reasons. First, it has a superior SNR and it also does not require an external reference, thus, reducing the hardware costs of the system.

2) Particle Filters: Particle filtering also implements the Bayes filter technique, but unlike the Kalman filter, it does not require the model to be linear or the distribution to be unimodal. In order to overcome the mathematical intractability of the Bayes method while incorporating multimodal distributions, a Monte Carlo sampling approach is implemented as described in [73]. In order to be capable of expressing the state changes, $N$ samples (particles) are randomly selected from the probability density function (pdf). These particles have weights proportional to the amplitude of the pdf at their sample point and the weights have the property that their sum is equal to 1 . Each particle is then passed through the process model to determine the next state of that particle. As a result, the particle filter becomes more computationally complex, but more accurate the more samples you choose, to the point where if $N=\infty$, then the system will give the optimal Bayesian solution. The weights of the new particles are determined using an update equation that incorporates the measurement model, and the estimated state is the mean of the determined pdf. One issue with the particle filter is that over time a small number of the particles become dominant, to the point where only a single particle will have a nonzero weighting. To prevent this from occurring, a number of different solutions have been described [74]. One of the most common techniques used to stop degeneracy is called the sampling-importance-resampling technique. Using this technique, if the number of effective particles is less than a given threshold, then the particles are sampled with replacement from the current batch of particles proportional to the weights of the particles. Once $N$ particles have been sampled the weights of each particle are reset to $1 / N$. 
These new particles are then passed through the process model to determine the new states and the algorithm repeats.

Little work has been done to date for artifact removal using the particle filter in physiological signals. However, artifact removal using the particle filter is similar to that accomplished using the Kalman filter. Process and measurement models are required prior to employing the algorithm. In 2010, Hongxia and Jifang [75] used the particle filter to remove noise from simulated and actual motor bearing vibration signals. This was accomplished by determining a nonlinear process model for the system and having the measurement model as the current state plus the added noise. Therefore, by determining the next state of the system, the algorithm is determining the best estimate of the signal without the added artifact.

Kalman filters require sensors with high accuracy levels and fast update rates to perform optimally. They are one of the most efficient filtering techniques, that use the Bayes' method, in relation to computation time and memory. However, if accurate sensors are not available, or if there is no detailed model of the process and measurements then particle filtering becomes the more viable option. Particle filters do not require a detailed model of the systems and, thus, are a very flexible tool with a relatively low implementation overhead [62].

\section{Blind Source Separation (BSS)}

BSS is based on a wide class of unsupervised learning algorithms with the goal of estimating sources (which are not necessarily independent) and parameters of a mixing system [76]

$$
\left[\begin{array}{c}
\mathbf{x}_{1} \\
\mathbf{x}_{2} \\
\vdots \\
\mathbf{x}_{P}
\end{array}\right]=\left[\begin{array}{cccc}
a_{11} & a_{12} & \cdots & a_{1(M+Q)} \\
a_{21} & a_{22} & \cdots & a_{2(M+Q)} \\
\vdots & \vdots & \ddots & \vdots \\
a_{P 1} & a_{P 2} & \cdots & a_{P(M+Q)}
\end{array}\right]\left[\begin{array}{c}
\mathbf{s}_{1} \\
\vdots \\
\mathbf{s}_{M} \\
\mathbf{v}_{1} \\
\vdots \\
\mathbf{v}_{Q}
\end{array}\right]+\mathbf{N}
$$

where $\mathbf{s}_{i}, \mathbf{v}_{i}, \mathbf{x}_{i} \in \mathbb{R}^{1 \times L}$ are row vectors of $L$ samples for the $i$ th original underlying source signal with specific statistical properties, the $i$ th contaminating artifact signal and the $i$ th recorded signal. $\mathbf{A}$ is the unknown mixing matrix and $\mathbf{N}$ is an unknown matrix representing noise and error values. For the remainder of this paper, the combined source and artifact signal matrix will be referred to as $\mathbf{S}$.

Estimation is performed using only the output signals from the BSS algorithm, the recorded sensor signals and some assumptions on the signal; therefore, no additional reference signal is required. Most BSS models can be expressed algebraically in terms of matrix factorization, given observation $\mathbf{X}$ perform the matrix factorization, $\mathbf{X}=\mathbf{A S}$. This is the general form of the equation and it can be expressed as equation 1 with $P=M=Q$ and $\mathbf{A}=\left[\mathbf{I}_{M} ; \mathbf{I}_{M}\right]$, where $\mathbf{I}_{M}$ is the $M \times M$ identity matrix. As only the observations $\mathbf{X}$ are known, the BSS technique is used to generate an unmixing matrix to determine the original sources.

$$
\hat{\mathbf{S}}=W X
$$

where $\hat{\mathbf{S}}$ is the estimation of the original sources (and artifact) and $\mathbf{W}$ is the unmixing matrix. Once the estimations of the original sources are known, the sources representing the artifact signals can be removed.

There are number of different algorithms available to perform BSS, including independent component analysis (ICA) and canonical correlation analysis (CCA) described in the following. Each algorithm performs matrix factorization with the aid of some particular assumptions about the signals, such as independence for ICA. The fundamental approaches underpinning source separation algorithms, as described in Choi et al. [76], are: 1) the use of higher order statistics (HOS) including skewness and kurtosis; 2) second-order statistics (SOS) including delayed correlations and spectral diversity; 3) SOS with nonstationarity; and 4) the use of the signal characteristics (time, frequency) to separate the sources.

The choice of the optimum algorithm to employ depends on a priori knowledge of the signal. Some of the most common BSS algorithms employed in research are described as follows.

1) ICA: ICA is a BSS technique [77] in which recorded, multichannel signals are separated into their independent constituent components or sources [78]. There are many assumptions that the sources and mixing must adhere to for ICA to be allowed to be employed, including linear mixing, square mixing and stationary mixing. These assumptions are clarified further by James and Hesse [79].

One of the major limitations of ICA is the requirement for the independent sources to be non-Gaussian. ICA can obtain an estimate $\hat{\mathbf{s}}$ of the sources $\mathbf{s}$ iff the sources are non-Gaussian. If a single independent component is Gaussian, it can be estimated as the residual that is leftover after extracting all other independent components, but ICA cannot account for more than one Gaussian component. Unfortunately, a component is generally not known to be Gaussian or non-Gaussian a priori. The data are commonly whitened prior to the computation of the ICA. This can be achieved with principle component analysis (PCA). By doing so, the correlation between the signals is canceled and the dimensionality of the data can often be reduced.

There are a number of different algorithms used to determine the unmixing matrix $\mathbf{W}$. Some of the most commonly employed include fast ICA [78], the Bell-Sejnowski algorithm [80], extended ICA [81], and JADE [82]. Fast ICA separates the sources by maximizing the magnitude of the kurtosis or by maximizing the negentropy. The Bell-Sejnowski algorithm uses negentropy to try and separate the sources. The extended ICA algorithm is similar to that proposed by Bell and Sejnowski, but allows for sources that have a negative kurtosis. Finally, the JADE algorithm maximizes the kurtosis of the pdf through a joint diagonalization of the fourth-order cumulants.

When the recorded signals have been passed though the unmixing matrix $\mathbf{W}$, the outputs are deemed to be the original independent sources. The sources considered to be artifacts can then be removed. The artifact signals can often be determined using heuristics such as signal shape, frequency, and amplitude [83]. The remaining sources are thus the original desired signal sources, and when put through the inverse of the 
unmixing matrix $\mathbf{W}^{-1}$, the resulting outputs are the artifactcorrected signals [84], [85], [86].

While propagation delays associated with physiological signals are generally small, they could potentially be sufficient to violate the ICA assumptions of instantaneous mixing of the sources. An extension to the ICA algorithm, known as Convolutive ICA (CICA), has been developed which takes into account both weighted and delayed contributions of sources in the generation of the observations [87]. In doing so, the coefficients of the unknown mixing matrix $\mathbf{A}$ are substituted by finite impulse response filters and the product term of (10) is replaced by the convolution operator $\star$. Milanesi et al. [88] used a frequency domain approach with the CICA algorithm for the removal of motion artifacts from ECG and illustratively showed it to provide improved results compared to basic ICA. The algorithm has also been shown to improve the separation of EEG components [89] compared to the ICA algorithm.

ICA, as a nonparametric algorithm, has a major advantage over some other parametric algorithms, such as adaptive filtering, in that no a priori information is required for the algorithm to function. Thus, no reference signals are required, reducing the number of sensors allowing for a more portable device. However, if a priori information is used in the model, the ICA algorithm becomes much more informative as a technique [79].

2) CCA: CCA [90] is another BSS method for separating a number of mixed or contaminated signals. The CCA method uses second-order statistics (SOS) to generate components derived from their uncorrelated nature. CCA solves the BSS problem by forcing the sources to be maximally autocorrelated and mutually uncorrelated [91]. By looking for uncorrelated components, the approach uses a weaker condition than statistical independence sought by the ICA algorithm. ICA does not take temporal correlations into account; thus, the samples can be arranged arbitrarily in time and the method will return the same solution. CCA addresses this point by being capable of finding uncorrelated components that, in addition, have maximum spatial or temporal correlation within each component. Details on the algorithm used to find the unmixing matrix $\mathbf{W}$ using CCA can be found in [92]. Artifact removal can then be introduced by setting the columns of the unmixing matrix, which represent the artifacts in the recording to zero when performing the reconstruction.

Borga and Knutsson [92] compared both CCA and fast ICA for the problem of separating five different EEG channels. Both methods had qualitatively the same results, but as CCA employs SOS, where ICA employs HOS, the CCA method is more computationally efficient. The authors repeated this test with fMRI data in [93] and again showed that the CCA algorithm performed better in terms of computational complexity by at least an order of magnitude while having similar qualitative results, thus, lending itself toward implementation in the personal healthcare domain. It is also noted that the CCA method always returns the same result when employed with a given dataset, this, however, is not true when using the ICA algorithm.

In 2006, Clercq et al. [91] used CCA to successfully remove muscle artifacts from EEG. This method was also shown on simulated data to outperform an ICA-based technique (JADE), where the overall relative root MSE (RRMSE) for CCA was on average 2.48 times lower than that achieved using ICA. This result was again documented by Gao et al. [94] in 2010. The possible reason for CCA's improved performance over the ICA method is due to the form of the muscle activity. As ICA employs statistical independence to separate the sources, it is able to isolate those artifacts with stereotyped scalp topographies into a single independent component. Muscle artifacts, however, involve the movement of a group of muscles, which do not have a stereotyped topography and, thus, ICA does not function correctly.

\section{E. Artifact Removal From Single-Channel Measurements}

In addition to some of the artifact removal techniques described earlier which can operate on single channels (adaptive filter, Wiener filter, Bayes filters), there are also a number of additional algorithms devised to operate on systems that have only a single channel. These algorithms are utilized more frequently in the personal healthcare domain due to the desire for low hardware costs and operational setup complexity. The algorithms assume the same model as given in (9), but the observations are no longer multidimensional, hence (9) reduces to

$$
\mathbf{x}=\mathbf{a S}+\mathbf{n}
$$

where $\mathbf{x}, \mathbf{n} \in \mathbb{R}^{1 \times L}, \mathbf{a} \in \mathbb{R}^{1 \times(M+Q)}$ and $\mathbf{S} \in \mathbb{R}^{(M+Q) \times L}$. As there are deemed to be more sources then recorded signals, the BSS methods described earlier can no longer operate. The developed algorithms use a modified form of the previously established methods (assuming the same signal properties) in conjunction with some new algorithms. Three of these methods are described as follows.

1) Single-Channel ICA: As stated in section IV-D1, the independent sources $\hat{\mathbf{s}}$ can only be separated from the recorded mixed signals $\mathbf{x}$ if the inverted matrix $\mathbf{W}$ of the mixing matrix $\mathbf{A}$ can be determined, i.e., $\hat{\mathbf{s}}=W x$. However, basic ICA does not function if there are more sources than sensors. In 2007, Davies and James [95] demonstrated that ICA can be implemented on single-channel signals if the PSD of the mixed signals is disjointed. If this is the case, the recorded time series is then fragmented into a sequence of contiguous blocks. With these new vector observations, the problem of single-channel ICA (SCICA) transforms into a multiple channel ICA problem. Using the original ICA method, each signal can be decomposed and reconstructed in the observation domain achieved through the implementation of the mixing and unmixing matrices as described by Davies and James [95]. This algorithm was shown to be able to extract a number of spikes, seizure components, and ocular artifacts from a recorded single-channel EEG signal.

2) Dynamical Embedding (DE): DE creates a series of delay vectors of the recorded signal and uses these vectors to create an embedded matrix, also known as a trajectory matrix, as observed in [96]. This matrix needs to be further decomposed so as to access the information required from the signal. There are a number of different methods used to decompose these matrices, two of which will be described in more detail later. 
James and Lowe [96] used fast ICA to deconstruct the embedding matrix created using single-channel recordings from both EEG and magnetoencephalogram (MEG) embedded with ocular and 50-Hz-hum artifact, into to its informative components. The fast ICA algorithm was chosen due to its ease of implementation and speed of operation. The fast ICA algorithm performs as stated in section IV-D1 and deconstructs the embedded matrix into $m$ ICs. The next step in the process is the selection of the ICs that are not a result of artifacts, which is not always a trivial task. With these ICs chosen, they are then projected back onto the measurement space in isolation, where the embedding is reversed, thus, producing the resultant single-channel signal free of the detected artifacts.

Teixeira et al. [97] used an alternative method to extract the desired information from the embedded matrix. In this paper, the authors used local singular spectrum analysis (local SSA) to extract high-amplitude and low-frequency artifacts from singlechannel EEG recordings. Similar columns of the trajectory matrix are grouped together using a clustering algorithm such as $k$-means creating a number of subtrajectory matrices. PCA is then used to determine each individual cluster's eigenvalues and eigenvectors. Denoising is achieved by projecting the data onto the eigenvectors corresponding to the $k$ largest eigenvalues, with the value of $k$ (which can be different for each cluster) determined using the minimum description length criterion [97]. After denoising, each subtrajectory matrix is reconstructed as described in [97]. Each column of the new subtrajectory matrices is then assigned to a column of a new trajectory matrix. The columns, to which they are assigned, are determined according to the columns of the original trajectory matrix used to create the subtrajectory matrix. Finally, the 1-D signal is regenerated by averaging over the entries of the corresponding descending diagonals of the reconstructed trajectory matrix. This technique, unlike conventional ICA methods, does not require any user input in the selection of components of the reconstruction, thus, allowing for fully automatic artifact removal. It is capable of removing artifacts such as line noise, baseline drift and eye and head movements, all common in the personal healthcare measurement domain [97].

3) Morphological Component Analysis (MCA): MCA operates by decomposing the recorded signal into components that have different morphological characteristics. Each component is sparsely represented in an overcomplete dictionary, which is comprised of a collection of waveforms, called atoms, which can be used to describe the different underlying signals. The overall signal is thus a linear combination of these individual atoms multiplied by their coefficient vectors. The basis pursuit denoise model [98] is then used to find the sparsest representation. Once this has been completed, the bases that represent the artifact signal(s) can be removed.

In [98], the authors used the MCA algorithm to remove artifacts originating from eye-brow raising, jaw clenching, swallowing, and eye blinks from a single-channel EEG recording. Three dictionaries were chosen to represent the EEG signal, namely the Daubechies wavelet, the discrete cosine transform (DCT), and the Dirac basis. These bases were chosen as they are capable of representing the different morphologies of the recorded
EEG and artifact [98]. Their results visually demonstrate that the MCA algorithm reduces the artifacts in the simulated contaminated EEG signal, while also producing an improved correlation with the true signal $(R=0.5485)$ over that produced using the stationary wavelet transform $(R=0.2925)$. The artifacts due to $60-\mathrm{Hz}$ hum are not removed using this method however, as this artifact is also represented using the DCT basis. This can be removed by post processing using basic filtering. The system can be improved by adding more applicable dictionaries to the already overcomplete dictionary. This method has also been applied in multichannel systems by Yong et al. [99].

4) Wavelet ICA (WICA): WICA utilizes both the wavelet transform and ICA in the removal of artifacts from the desired signal. The wavelet transform has been used previously to remove artifacts from biomedical signals [100], [101], [102]. The discrete wavelet transform (DWT) is unable to remove artifacts which overlap in the spectral domain, for example, ECG artifact embedded on an EMG signal. ICA also has limitations as described earlier in Section IV-D1. One main assumption and limitation is the requirement that the number of sources is equal to the number of measurement sensors. The method ofWICA, as described in [12], combines the positive aspects of both independent algorithms to overcome some of their individual shortcomings. WICA can be used for single-channel recordings due to the wavelets creating multidimensional data from a single-channel measurement as described by Lin and Zhang in 2005 [103]. The DWT is first applied to the single-channel recording. The resulting wavelet resolutions are analyzed and the resolutions that contain artifacts components are manually selected. These artifact corrupted wavelet resolutions are then used to create a new dataset, which is used as the input to the chosen ICA algorithm. ICA is applied as specified in Section IVD1 and the components determined to be artifacts are removed. Wavelet reconstruction is then performed using both the unselected coefficients and the coefficients cleaned during the ICA step. This produces the multichannel signals with the artifact removed. Mijović et al. [104] demonstrated how this approach can be used to remove ECG artifacts from contaminated EMG recordings.

5) Empirical Mode Decomposition ICA (EMD-ICA): The EMD method was first described in 1998 [105] as a technique for nonlinear signal processing and is well suited to nonstationary signals. The EMD algorithm decomposes the signal into components called intrinsic mode functions (IMF) with welldefined instantaneous frequencies. These IMFs can then be used as inputs to an ICA algorithm and the mixing and unmixing matrices can be found [104]. The IC's corresponding to the artifact can be removed and the original signal minus the artifact can be obtained by adding all the new derived IMFs.

Ensemble EMD-ICA (EEMD-ICA) was tested against WICA and SCICA as a method for the removal of simulated artifacts from recorded physiological signals [104]. EEMD-ICA was seen to perform slightly better than the WICA algorithm (RRMSE ranging from $50.73 \%$ to $64.79 \%$ for EEMD-ICA and $\mathrm{RRMSE}=66.22 \%$ for WICA) at removing the artifacts from EMG and EEG data, with both outperforming the SCICA algorithm. As most biological signals are nonstationary and 
TABLE I

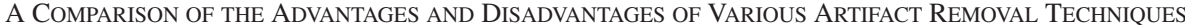

\begin{tabular}{|c|c|c|c|c|c|c|}
\hline & 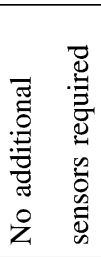 & 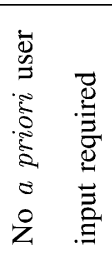 & 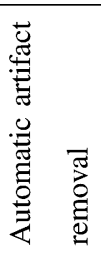 & 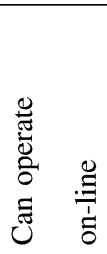 & 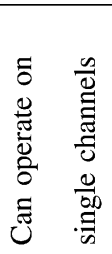 & 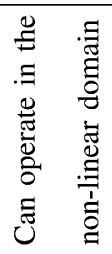 \\
\hline Adaptive filter & $x$ & $x$ & $\checkmark$ & $\checkmark$ & $\checkmark$ & $\checkmark$ \\
\hline Wiener filter & $\checkmark$ & $x$ & $\checkmark$ & $x$ & $\checkmark$ & $\checkmark$ \\
\hline Kalman filter & $\checkmark$ & $x$ & $\checkmark$ & $\checkmark$ & $\checkmark$ & $\checkmark$ \\
\hline Particle filter & $\checkmark$ & $x$ & $\checkmark$ & $\checkmark$ & $\checkmark$ & $\checkmark$ \\
\hline Independent Component Analysis & $\checkmark$ & $\checkmark$ & $x$ & $\checkmark$ & $x$ & $\checkmark$ \\
\hline Canonical Correlation Analysis & $\checkmark$ & $\checkmark$ & $x$ & $x$ & $x$ & $\checkmark$ \\
\hline Single Channel ICA & $\checkmark$ & $\checkmark$ & $x$ & $x$ & $\checkmark$ & $\checkmark$ \\
\hline Dynamical Embedding ICA & $\checkmark$ & $\checkmark$ & $x$ & $x$ & $\checkmark$ & $\checkmark$ \\
\hline Dynamical Embedding SSA & $\checkmark$ & $\checkmark$ & $\checkmark$ & $x$ & $\checkmark$ & $x$ \\
\hline Morphological Component Analysis & $\checkmark$ & $x$ & $\checkmark$ & $x$ & $\checkmark$ & $x$ \\
\hline Wavelet ICA & $\checkmark$ & $\checkmark$ & $x$ & $x$ & $\checkmark$ & $\checkmark$ \\
\hline Empirical Mode Decomposition ICA & $\checkmark$ & $\checkmark$ & $x$ & $x$ & $\checkmark$ & $\checkmark$ \\
\hline
\end{tabular}

nonlinear, the EEMD-ICA algorithm adapts itself effectively to real world situations.

\section{DISCUSSION}

The algorithms examined earlier are among the most commonly implemented in the biomedical signal processing field. The choice of which algorithm to employ depends on a number of different factors, some of which are discussed later.

Long-term patient monitoring is often required to evaluate a patients well being and is commonly performed in an out-patient domain. This requires that the systems implemented are capable of operating for prolonged periods, without the overhead of frequent changing or charging of batteries. The computational cost of the chosen artifact removal algorithm therefore becomes an important concern. If significant power is consumed by onboard processing, battery life will reduce and this may require excessive changing or charging of the batteries, thus, disrupting the desired recordings. It is also possible to perform long-term recordings and to implement the artifact removal on a PC offline. In these situations, the computational load of the algorithm becomes less problematic, but the results are no longer reported in real time.

The availability of reference signals should also be taken into account when designing or selecting an algorithm. If available, simple adaptive filter algorithms can be used, with a low computational cost. They can also improve the accuracy and robustness of techniques, such as ICA [79]. Reference signals for the artifact can also be employed to determine the epochs of data, where artifact removal techniques should be administered [41]. The reference data specify the samples in the recorded signals, where artifacts are likely to be present, thus, manipulating this information can reduce the computational cost of the system.

The number and quality of recording sensors also has an impact on the choice of artifact removal technique employed. It is a requirement in most BSS algorithms that there be as many measurement sensors as underlying sources. In the personal healthcare environment, there is often a reduction in the number of sensors with respect to the clinical recording setting, due to the complexity or time required during setup. Therefore, algorithms utilized in these environments are required to be capable of separating sources with no redundant sensors as described in section IV-E. A reduction in the quality of the measurement sensors, commonly observed outside the clinical setting, can often necessitate the use of more computationally expensive artifact removal techniques, for example, from the Kalman filter to the particle filter. These techniques do, however, require accurate models of the signals, which may not always be available.

Although a variety of algorithms have been developed for removing unwanted artifacts, artifact removal continues to be an open research problem. As evident from Table I, there is currently no single best method that is efficient and robust over a wide range of conditions. Therefore, in the future, custom algorithms based on circumstances are likely to develop. Some current algorithms, such as EEMD-ICA, incorporate many of the previously mentioned desired attributes, but fail to satisfy them all. It is felt that in the coming years the joint use of a number of new and currently available methods will allow for all the attributes to be fulfilled. For example, the use of EEMD-CCA may allow for similar results to those achieved using EEMDICA; however, there will be a significant drop in computational complexity due to the use of a second-order algorithm, CCA. Further, as hardware and signal processing techniques continue to improve in computational resource and cost, respectively, the artifact removal systems will continue to be implemented more readily in nonclinical settings, increasing the comfort levels for the users while reducing the deployment costs [3].

\section{REFERENCES}

[1] Gartner-Inc. (2009, Jul.). "eHealth for a healthier europe," [Online]. Available: http://www.sweden.gov.se/sb/d/574/a/129815 
[2] Office of the Actuary. (2010, Jan.). "National Health Care Expenditures Data," [Online]. Available: www.hhs.gov

[3] E. Waterhouse, "New horizons in ambulatory electroencephalography," IEEE Eng. Med. Biol. Mag., vol. 22, no. 3, pp. 74-80, May/Jun. 2003.

[4] D. F. Evans, G. Pye, R. Bramley, A. G. Clark, T. J. Dyson, and J. D. Hardcastle, "Measurement of gastrointestinal $\mathrm{pH}$ profiles in normal ambulant human subjects," Gut, vol. 29, no. 8, pp. 1035-1041, 1988.

[5] C. Tsai, Y. Chen, "Development and testing of a perspiration measuring system," Med. Eng. Phys., vol. 32, pp. 356-362, 2010.

[6] L. Sornmo and P. Laguna, Electrocardiogram (ECG) Signal Processing, vol. 2, M. Akay, Ed. New York: Wiley, 2006.

[7] D. Z. Kowey and P R. Kocovic, Ambulatory Electrocardiographic Recording. Dallas, TX: American Heart Association, 2003.

[8] D. Jabaudon, J. Sztajzel, K. Sievert, T. Landis, and R. Sztajzel, Usefulness of Ambulatory 7-Day ECG Monitoring for the Detection of Atrial Fibrillation and Flutter After Acute Stroke and Transient Ischemic Attack. Dallas, TX: American Heart Association, 2004.

[9] A. C. Guyton and J. E. Hall, Textbook of Medical Physiology. New York: Elsevier Saunders, 2006.

[10] G. Robertson, G. Caldwell, J. Hamill, G. Kamen, and S. Whittlesey, Research Methods in Biomechanics. Champaign, IL: Human Kinetics, 2004.

[11] M. Prahlow, N. D., M. Buschbacher, and R. M., "An Introduction to electromyography: An invited review," J. Long-Term Effects Med. Implants, vol. 13, 2003.

[12] B. Azzerboni, M. Carpentieri, F. La Foresta, and F. Morabito, "NeuralICA and wavelet transform for artifacts removal in surface EMG," in Proc. IEEE Int. Joint Conf. Neural Netw., Jul.25-29, 2004, vol. 4, pp. 32233228.

[13] A. Chhikara, A. S. C. Rice, A. H. McGregor, and F. Bello, "Wearable device for monitoring disability associated with low back pain," in Proc. Int. Workshop Wearable Micro Nanosyst. Personal. Health, 2008.

[14] J. S. Karlsson, U. Wiklund, L. Berglin, N. Östlund, M. Karlsson, T. Bäcklund, K. Lindecrantz, and L. Sandsjö, "Wireless Monitoring of Heart Rate and Electromyographic Signals using a Smart T-shirt," in Proc. Int. Workshop Wearable Micro Nanosyst. Personal. Health, 2008.

[15] M. H. Boostani, R. Moradi, "Evaluation of the forearm EMG signal features for the control of a prosthetic hand," Physiol. Meas., vol. 24, no. 2, p. 309, 2003.

[16] Y. Su, A. Wolczowski, M. Fisher, G. Bell, D. Burn, and R. Gao, "Towards an EMG Controlled Prosthetic Hand Using a 3D Electromagnetic Positioning System," in Proc. Instrum. Meas. Technol. Conf., May, 2005, vol. 1, pp. 261-266.

[17] E. Niedermeyer and F. Lopes da Silva, in Electroencephalography: Basic Principles, Clinical Applications and Related Fields, C. Mitchel, Ed. Baltimore, MD: Williams \& Wilkins, 1999.

[18] A. Shoeb, S. Schachter, D. Schomer, B. Bourgeois, S. Treves, and J. Guttag, "Detecting seizure onset in the ambulatory setting: Demonstrating feasibility," in Proc. 27th EMBS Annu. Int. Conf., Jan. 2005, pp. 35463550.

[19] C. Berka, D. J. Levendowski, P. Westbrook, G. Davis, M. N. Lumicao, R. E. Olmstead, M. Popovic, V. T. Zivkovic, and C. K. Ramsey, "EEG quantification of alertness: Methods for early identification of individuals most susceptible to sleep deprivation," vol. 5797, pp. 78-89, May 2005.

[20] C. Berka, D. J. Levendowski, M. M. Cvetinovic, M. M. Petrovic, G. Davis, M. N. Lumicao, V. T. Zivkovic, M. V. Popovic, and R. Olmstead, "Realtime analysis of EEG indexes of alertness, cognition, and memory acquired with a wireless EEG headset," Int. J. Human-Comput. Interact., vol. 17, pp. 151-170, 2004.

[21] C. Papadelis, C. Kourtidou-Papadeli, P. Bamidis, I. Chouvarda, D. Koufogiannis, E. Bekiaris, and N. Maglaveras, "Indicators of sleepiness in an ambulatory EEG study of night driving," in Proc. 28th IEEE EMBS Annu. Int. Conf., Aug. 30-Sep. 3, 2006, pp. 6201-6204.

[22] R. Stevens, T. Galloway, and C. Berka, "Integrating EEG models of cognitive load with machine learning models of scientific problem solving." 2006.

[23] C. J. Bell, P. Shenoy, R. Chalodhorn, and R. P. N. Rao, "Control of a humanoid robot by a noninvasive brain-computer interface in humans," J. Neural Eng., vol. 5, no. 2, pp. 214-220, 2008.

[24] J. C. Lee, "Using a low-cost electroencephalograph for task classification in HCI Research," in Proc. UIST, 2006, pp. 81-90.

[25] T. Sullivan, S. Deiss, T.-P. Jung, and G. Cauwenberghs, "A brain-machine interface using dry-contact, low-noise EEG sensors," in Proc. IEEE Int. Symp. Circuits Syst., May, 2008, pp. 1986-1989.
[26] J. R. Wolpaw and D. J. McFarland, "Control of a two-dimensional movement signal by a noninvasive brain-computer interface in humans," in Proc. Natl. Acad. Sci., U.S.A, 2004, vol. 101, no. 51, pp. 17849-17854.

[27] M. Cope, "The application of near infrared spectroscopy to non invasive monitoring of cerebral oxygenation in the newborn infant" $\mathrm{Ph} . \mathrm{D}$. dissertation, Univ. London, 1991.

[28] K. H. Shelley, D. H. Jablonka, A. A. Awad, R. G. Stout, H. Rezkanna, and D. G. Silverman, "What is the best site for measuring the effect of ventilation on the pulse oximeter waveform?," Anesthesia Analgesia, vol. 103, no. 2, pp. 372-377, 2006.

[29] J. P. Phillips, P. A. Kyriacou, D. P. Jones, K. H. Shelley, and R. M. Langford, "Pulse oximetry and photoplethysmographic waveform analysis of the esophagus and bowel," Curr. Opin. Anesthesiol., vol. 21, pp. 779-783, 2008.

[30] R. T. Brouillette, A. Morielli, A. Leimanis, K. A. Waters, R. Luciano, and F. M. Ducharme, "Nocturnal pulse oximetry as an abbreviated testing modality for pediatric obstructive sleep apnea," Pediatrics, vol. 105, no. 2, pp. 405-412, 2000.

[31] S. Bunce, M. Izzetoglu, K. Izzetoglu, B. Onaral, and K. Pourrezaei, "Functional near-infrared spectroscopy," IEEE Eng. Med. Biol. Mag., vol. 25, no. 4, pp. 54-62, Jul./Aug. 2006.

[32] T. J. Huppert, S. G. Diamond, M. A. Franceschini, and D. A. Boas, "HomER: A review of time-series analysis methods for near-infrared spectroscopy of the brain," Appl. Opt., vol. 48, no. 10, pp. D280-D298, 2009.

[33] K. Izzetoglu, S. Bunce, B. Onaral, K. Pourrezaei, and B. Chance, "Functional optical brain imaging using near-infrared during cognitive tasks," Int. J. Human-Comput. Interact., vol. 17, pp. 211-227, 2004.

[34] F. Matthews, B. Pearlmutter, T. Ward, C. Soraghan, and C. Markham, "Hemodynamics for brain-computer interfaces," IEEE Signal Process. Mag., vol. 25, no. 1, pp. 87-94, 2008.

[35] F. Fanfulla, Mortara., R. Maestri, G. Pinna, C. Bruschi, F. Cobelli, and C. Rampulla, "The development of hyperventilation in patients with chronic heart failure and Cheyne-Stokes respiration," Chest, vol. 114, pp. 1083-1090, 1998.

[36] C. Tantucci, P. Bottini, C. Fiorani, M. Dottorini, F. Santeusanio, L. Provinciali, C. Sorbini, and G. Casucci, "Cerebrovascular reactivity and hypercapnic respiratory drive in diabetic autonomic neuropathy," J. Appl. Physiol., vol. 90, pp. 889-896, 2001.

[37] S. Bell, M. Saunders, J. Elborn, and D. Shale, "Resting energy expenditure and oxygen cost of breathing in patients with cystic fibrosis," Thorax, vol. 51, pp. 126-131, 1996.

[38] W. W. Flemons, M. R. Littner, J. A. Rowley, P. Gay, W. M. Anderson, D. W. Hudgel, R. D. McEvoy, and D. I. Loube, "Home Diagnosis of Sleep Apnea: A Systematic Review of the Literature," Chest, vol. 124, no. 4, pp. 1543-1579, 2003.

[39] S. C. Tarrant, R. E. Ellis, F. C. Flack, and W. G. Selley, "Comparative review of techniques for recording respiratory events at rest and during deglutition," Dysphagia, vol. 12, pp. 24-38, 1997.

[40] M. A. C. van Rijn, A. Peper, and C. A. Grimbergen, "High-quality recording of bioelectric events-Part 1: Interference reduction, theory and practice," Med. Biol. Eng. Comput., vol. 28, no. 5, pp. 389-97, Sep. 1990.

[41] K. Sweeney, D. Leamy, T. Ward, and S. McLoone, "Intelligent artifact classification for ambulatory physiological signals," in Proc. IEEE Anпи. Int. Conf. Eng. Med.Biol. Soc. (EMBC), Aug. 31-Sep. 4, 2010, pp. 63496352.

[42] K. Sweeney, T. Ward, and S. McLoone, "A simple bio-signals quality measure for in-home monitoring," in Proc. 7th IASTED Int. Conf., Innsbruck, Austria, Feb. 2010.

[43] E. Huigen, A. Peper, and C. A. Grimbergen, "Investigation into the origin of the noise of surface electrodes," Med. Biol. Eng. Comput, vol. 40, pp. 332-338, 2002.

[44] N. Thakor and Y.-S. Zhu, "Applications of adaptive filtering to ECG analysis: noise cancellation and arrhythmia detection," IEEE Trans. Biomed. Eng., vol. 38, no. 8, pp. 785-794, Aug. 1991.

[45] P. He, G. Wilson, and C. Russell, "Removal of ocular artifacts from electroencephalogram by adaptive filtering," Med. Biol. Eng. Comput., vol. 42, pp. 407-412, 2004.

[46] J. N. Knight, "Signal fraction analysis and artifact removal in EEG," Master's thesis, Department Comput. Sci., Colorado State Univ., 2003.

[47] V. S. Frost, J. A. Stiles, K. S. Shanmugan, and J. C. Holtzman, "A model for radar images and its application to adaptive digital filtering of multiplicative noise," IEEE Trans. Pattern Anal. Mach. Intell., vol. PAMI-4, no. 2, pp. 157-166, Mar. 1982.

[48] D. G. Manolakis, V. K. Ingle, and S. M. Kogon, Statistical and Adaptive Signal Processing. New York: McGraw-Hill, 1999. 
[49] G. Comtois and Y. Mendelson, "A noise reference input to an adaptive filter algorithm for signal processing in a wearable pulse oximeter," in Proc. IEEE 33rd Annu. Northeast Bioeng. Conf., Mar., 2007, pp. 106107.

[50] M. Milanesi, N. Martini, N. Vanello, V. Positano, M. Santarelli, R. Paradiso, D. De Rossi, and L. Landini, "Multichannel techniques for motion artifacts removal from electrocardiographic signals," in Proc. IEEE 28th Eng. Med. Biol. Soc. Annu. Int. Conf., Aug. 30-Sep. 3 2006, pp. 33913394.

[51] D. Tong, K. Bartels, and K. Honeyager, "Adaptive reduction of motion artifact in the electrocardiogram," in Proc. Second Joint 24th Annu. EMBS/BMES Conf., 2002, vol. 2, pp. 1403-1404.

[52] P. Hamilton, M. Curley, R. Aimi, and C. Sae-Hau, "Comparison of methods for adaptive removal of motion artifact," in Proc. Comput. Cardiol., 2000, pp. 383-386.

[53] P. Hamilton and M. Curley, "Adaptive removal of motion artifact [ECG recordings]," in Proc.IEEE 19th Eng. Med. Biol. Soc. Annu. Int. Conf., Oct. 2-Nov, 1997, vol. 1, pp. 297-299.

[54] M. Zia Ur Rahman, R. A. Shaik, and D. V. R. K. Reddy, "Cancellation of artifacts in ECG Signals using sign-based normalized adaptive filtering technique," in Proc. IEEE Symp. Ind. Electron. Appl., Oct.4-6, 2009, vol. 1, pp. 442-445.

[55] M. Arezki, A. Benallal, and D. Berkani, "Improvement of the simplified fast transversal filter type algorithm for adaptive filtering," J. Comput. Sci., vol. 5, no. 5, pp. 347-354, 2010 .

[56] D. L. Jones, S. Appadwedula, M. Berry, M. Haun, D. Moussa, and D. Sachs, “Adaptive Filtering: LMS Algorithm,” Tech. Rep., 2009.

[57] T. Benesty, J. Gansler, "New insights into the RLS algorithm," EURASIP J. Appl. Signal Process., pp. 331-339, 2004.

[58] C. Marque, C. Bisch, R. Dantas, S. Elayoubi, V. Brosse, and C. Pérot, "Adaptive filtering for ECG rejection from surface EMG recordings," $J$. Electromyogr. Kinesiol., vol. 15, no. 3, pp. 310-315, 2005.

[59] M. Raya and L. Sison, "Adaptive noise cancelling of motion artifact in stress ECG signals using accelerometer," in Proc. Second Joint 24th EMBS/BMES Annu. Conf., vol. 2, pp. 1756-1757.

[60] P. Diniz, Adaptive Filtering: Algorithms and Practical Implementation. New York: Springer, 2008

[61] M. Izzetoglu, A. Devaraj, S. Bunce, and B. Onaral, "Motion artifact cancellation in NIR spectroscopy using Wiener filtering," IEEE Trans. Biomed. Eng., vol. 52, no. 5, pp. 934-938, May 2005.

[62] D. Fox, J. Hightower, L. Liao, D. Schulz, and G. Borriello, "Bayesian filtering for location estimation," IEEE Pervas. Comput., vol. 2, no. 3, pp. 24-33, Jul./Sep. 2003.

[63] R. E. Kalman, "A new approach to linear filt ering and prediction problems," Trans. ASME, J. Basic Eng. Series D, vol. 82, pp. 35-45, 1960.

[64] G. Welch and G. Bishop, "An introduction to the Kalman filter," Univ. North Carolina at Chapel Hill, Chapel Hill, Tech. Rep. TR 95-041, 2006.

[65] M. Izzetoglu, P. Chitrapu, S. Bunce, and B. Onaral, "Motion artifact cancellation in NIR spectroscopy using discrete Kalman filtering," BioMed. Eng. Online, vol. 9, no. 1, p. 16, 2010.

[66] S. Seyedtabaii and L. Seyedtabaii, "Kalman filter based adaptive reduction of motion artifact from photoplethysmographic signal," World Acad. Sci., Eng. Technol., vol. 37, 2008.

[67] F. Morbidi, A. Garulli, D. Prattichizzo, C. Rizzo, and S. Rossi, "Application of Kalman filter to remove TMS-induced artifacts from EEG recordings," IEEE Trans. Control Syst. Technol., vol. 16, no. 6, pp. 1360-1366, Nov. 2008.

[68] K. Rheinberger, T. Steinberger, K. Unterkofler, M. Baubin, A. Klotz, and A. Amann, "Removal of CPR artifacts from the ventricular fibrillation ECG by adaptive regression on lagged reference signals," IEEE Trans. Biomed. Eng., vol. 55, no. 1, pp. 130-137, Jan. 2008.

[69] G. Bonmassar, P. L. Purdon, I. P. Jskelinen, K. Chiappa, V. Solo, E. N. Brown, and J. W. Belliveau. (2002). Motion and ballistocardiogram artifact removal for interleaved recording of eeg and eps during MRI. NeuroImage [Online]. 16(4), pp. 1127-1141. Available: http://www.sciencedirect.com/science/article/pii/S1053811902911250

[70] M. Rohalova, P. Sykacek, M. Koska, and G. Dorffner, "Detection of the EEG Artifacts by the Means of the (Extended) Kalman Filter," Meas. Sci. Rev., vol. 1, pp. 59-62, 2001.

[71] R. Sameni, M. Shamsollahi, C. Jutten, and M. Babaie-Zadeh, "Filtering noisy ECG signals using the extended Kalman filter based on a modified dynamic ECG model," in Proc. Comput. Cardiol., Sep. 25-28, 2005, pp. 1017-1020.

[72] R. Sameni, M. Shamsollahi, C. Jutten, and G. Clifford, "A nonlinear bayesian filtering framework for ecg denoising," IEEE Trans. Biomed. Eng., vol. 54, no. 12, pp. 2172-2185, 2007.
[73] A. Doucet, N. De Freitas, and N. Gordon, Sequential Monte Carlo Methods in Practice (Statistics for Engineering and Information Science), A Doucet, N. De Freitas, and N. Gordon, Eds. New York: Springer-Verlag, 2001.

[74] J. V. Candy. (2009, Apr.). Bayesian Signal Processing: Classical, Modern and Particle Filtering Methods (Adaptive and Learning Systems for Signal Processing, Communications and Control Series). New York: Wiley-Interscience, [Online]. Available: http://www.worldcat.org/isbn/9780470180945

[75] P. Hongxia and M. Jifang, "De-noising method research on bearing fault signal based on particle filter," in Proc. Second World Congr. Nature Biol. Inspired Comput., Dec., 2010, pp. 311-315.

[76] S. Choi, A. Cichocki, H. min Park, and S. young Lee, "Review blind source separation and independent component analysis: A review," Neural Inf. Process., Lett. Rev., vol. 6, no. 1, pp. 1-57, 2004.

[77] P. Comon, "Independent component analysis, a new concept?," Signal Process., vol. 36, no. 3, pp. 287-314, 1994.

[78] A. Hyvärinen, J. Karhunen, and E. Oja, Independent Component Analysis, S. Haykin, Ed. New York: Wiley-Interscience, Jun. 2001.

[79] C. J. James and C. W. Hesse, "Independent component analysis for biomedical signals," Physiol. Meas., vol. 26, pp. 15-39, 2005.

[80] A. J. Bell and T. J. Sejnowski, "An information-maximization approach to blind separation and blind deconvolution," Neural Comput., vol. 7, pp. 1129-1159, 1995.

[81] T.-W. Lee, M. Girolami, and T. J. Sejnowski, "Independent componen analysis using an extended infomax algorithm for mixed subgaussian and supergaussian sources," Neural Comput., vol. 11, no. 2, pp. 417-441, 1999.

[82] B. A. Draper, K. Baek, M. S. Bartlett, and J. R. Beveridge, "Recognizing faces with PCA and ICA," in Comput. Vis. Image Understanding (Special Issue on Face Recognition), 2003, pp. 115-137.

[83] G. Gomez-Herrero, W. De Clercq, H. Anwar, O. Kara, K. Egiazarian, S. Van Huffel, and W. Van Paesschen, "Automatic removal of ocular artifacts in the EEG without an EOG reference channel," in Proc. 7th Nordic Signal Process. Symp., Jun. 2006, pp. 130-133.

[84] R. Li and J. C. Principe. (2006). "Blinking artifact removal in cognitive EEG data using ICA," in Proc. IEEE Int. Conf. Eng. Med. Biol. Soc. [Online]. 1(3), pp. 5273-5276. Available: http://www.ncbi.nlm.nih.gov/pubmed/17946690

[85] T.-P. Jung, S. Makeig, C. Humphries, T.-W. Lee, M. J. McKeown, V. Iragui, and T. J. Sejnowski, "Removing electroencephalographic artifacts by blind source separation," Psychophysiology, vol. 37, no. 02, pp. 163-178, 2000.

[86] M. Chawla, H. Verma, and V. Kumar, "Artifacts and noise removal in electrocardiograms using independent component analysis," Int. J. Cardiol., vol. 129, no. 2, pp. 278-281, 2008.

[87] C. Vaya, J. Rieta, C. Sanchez, and D. Moratal, "Convolutive blind source separation algorithms applied to the electrocardiogram of atrial fibrillation: Study of performance," IEEE Trans. Biomed. Eng., vol. 54, no. 8, pp. 1530-1533, Aug. 2007.

[88] M. N. Milanesi, N. Martini, V. Vanello, P. M. F, Santarelli, and L. Landini, "Independent component analysis applied to the removal of motion artifacts from electrocardiographic signals," Med.Biol.Eng. Comput., vol. 46, no. 3, pp. 251-261, Mar. 2008.

[89] A. Yamazaki, T. Tajima, and K. Matsuoka, "Convolutive independent component analysis of EEG data," in Proc. Annu. Conf. SICE, Aug., 2003, vol. 2, pp. 1227-1231.

[90] H. Hotelling, "Relations between two sets of variates," Biometrika, vol. 28, no. 3-4, pp. 321-377, 1936.

[91] W. D. Clercq, A. Vergult, B. Vanrumste, W. Van Paesschen, and S. Van Huffel, "Canonical correlation analysis applied to remove muscle artifacts from the electroencephalogram," IEEE Trans. Biomed. Eng., vol. 53, no. 12 , pp. 2583-2587, Nov. 2006.

[92] M. Borga and H. Knutsson, "A canonical correlation approach to blind source separation,” Dept. Biomedical Eng., Linköping Univ., Linkoping, Sweden, Tech. Rep. LiU-IMT-EX-0062, 2001.

[93] M. Borga, O. Friman, P. Lundberg, and H. Knutsson, "A Canonical Correlation Approach to Exploratory Data Analysis in fMRI," presented at the ISMRM Annu. Meet., Honolulu, Hawaii, May 2002.

[94] J. Gao, C. Zheng, and P. Wang, "Online removal of muscle artifact from electroencephalogram signals based on canonical correlation analysis," Clin. EEG Neurosci., vol. 41, no. 1, p. 53, 2010.

[95] M. Davies and C. James, "Source separation using single channel ICA," Signal Process., vol. 87, no. 8, pp. 1819-1832, 2007.

[96] C. J. James and D. Lowe, "Extracting multisource brain activity from a single electromagnetic channel," Artif. Intell. Med., vol. 28, no. 1, pp. 89104, 2003. 
[97] A. Teixeira, A. Tome, E. Lang, P. Gruber, and A. Martins da Silva, "Automatic removal of high-amplitude artefacts from single-channel electroencephalograms.," Comput. Methods Programs Biomed., vol. 83, pp. 125138, 2006.

[98] X. Yong, R. Ward, and G. Birch, "Artifact removal in EEG using Morphological Component Analysis," in Proc. IEEE Int. Conf. Acoust., Speech Signal Process., Apr.19-24, 2009, pp. 345-348.

[99] X. Yong, R. Ward, and G. Birch, "Generalized Morphological Component Analysis for EEG source separation and artifact removal," in Proc. 4th Int. IEEE/EMBS Conf. Neural Eng., Apr. 2009, pp. 343-346.

[100] P. S. Kumar, R. Arumuganathan, K. Sivakumar, and C. Vimal, "Removal of Ocular Artifacts in the EEG through Wavelet Transform without using an EOG Reference Channel," Int. J. Open Problems Comput. Math., vol. 1,2008

[101] O. A. Rosso, A. Figliola, J. Creso, and E. Serrano, "Analysis of waveletfiltered tonic-clonic electroencephalogram recordings," Med. Biol. Eng. Comput., vol. 42, pp. 516-523, 2004.

[102] H. Liang and Z. Lin, "Stimulus artifact cancellation in the serosal recordings of gastric myoelectric activity using wavelet transform," IEEE Trans. Biomed. Eng., vol. 49, no. 7, pp. 681-688, Jul. 2002.

[103] J. Lin and A. Zhang, "Fault feature separation using wavelet-ICA filter," Int. Nondestructive Testing Eval., vol. 38, no. 6, pp. 421-427, 2005.

[104] B. Mijović, M. De Vos, I. Gligorijevic and, J. Taelman, and S. Van Huffel, "Source separation from single-channel recordings by combining empirical-mode decomposition and independent component analysis," IEEE Trans. Biomed. Eng., vol. 57, no. 9, pp. 2188-2196, Sep. 2010.

[105] N. E. Huang, Z. Shen, S. R. Long, M. C. Wu, H. H. Shih, Q. Zheng, N. C. Yen, C. C. Tung, and H. H. Liu, "The empirical mode decomposition and the hilbert spectrum for nonlinear and non-stationary time series analysis," in Proc. R. Soc. Lond. Series A: Math., Phys. Eng. Sci., vol. 454, no. 1971, pp. 903-995, Mar. 1998.

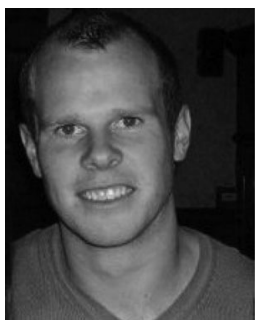

Kevin T. Sweeney (S'09) was born in Co. Meath, Ireland in 1984. He received a bachelor degree in electronic engineering with first class honors from the National University of Ireland (NUI), Maynooth, Ireland, in 2006, where he is currently working toward the $\mathrm{Ph} . \mathrm{D}$ degree in biomedical engineering.

His current research interests include biomedical signal processing specializing in the removal of motion artifacts from physiological signals.

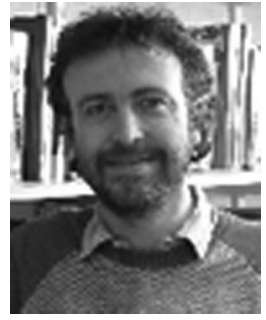

Tomás E. Ward (S'94-M'99-SM'12) received the B.E. degree in electronic, and the M.Eng.Sc and Ph.D degrees in biomedical engineering from University College, Dublin, Ireland in 1994, 1996, and 1999, respectively.

In 1999, he was appointed as a Lecturer in the Department of Computer Science at the National University of Ireland (NUI), Maynooth, Ireland. In 2000, he was appointed as a Lecturer in the Department of Electronic Engineering, NUI, where he is currently a Senior Lecturer. His primary research areas include the application of brain computer interfaces for neurorehabilitation particularly in stroke, closed-loop communication protocols for distributed interactive applications, and virtual environments for cognitive and physical rehabilitation.

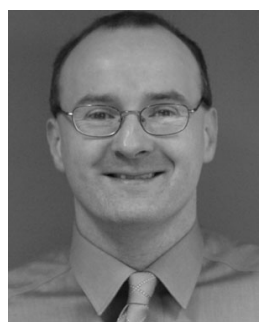

Seán F. McLoone (S'94-M'96-SM'02) received the M.E. degree (with distinction) in electrical and electronic engineering, and the Ph.D. degree in control engineering in 1992 and 1996, respectively, from Queens University, Belfast, U.K.

$\mathrm{He}$ is currently a Senior Lecturer and Head of the Department of Electronic Engineering, National University of Ireland, Maynooth, Ireland. His research interests are focused on the general area of data-based modelling and analysis of dynamical systems. This encompasses techniques ranging from classical system identification, fault diagnosis and statistical process control to modern artificial intelligence inspired adaptive learning algorithms and optimization techniques. His current research interests include data analysis and signal processing for in-home health monitoring and assisted living technologies, algorithms for unsupervised sparse feature selection, and clustering with application to large datasets. 